ESAIM: PROCEEDINGS, March 2012, Vol. 35, p. 275-280

Fédération Denis Poisson (Orléans-Tours) et E. Trélat (UPMC), Editors

\title{
PARALLEL COMPUTING FOR THE STUDY OF THE FOCUSING DAVEY-STEWARTSON II EQUATION IN SEMICLASSICAL LIMIT*
}

\author{
KRISTELLE ROIDOT ${ }^{1}$
}

\begin{abstract}
The asymptotic description of the semiclassical limit of nonlinear Schrödinger equations is a major challenge with so far only scattered results in $1+1$ dimensions. In this limit, solutions to the NLS equations can have zones of rapid modulated oscillations or blow up. We numerically study in this work the Davey-Stewartson system, a $2+1$ dimensional nonlinear Schrödinger equation with a nonlocal term, by using parallel computing. This leads to the first results on the semiclassical limit for the Davey-Stewartson equations.
\end{abstract}

\section{INTRODUCTION}

Nonlinear Schrödinger equations (NLS) are equations of the form

$$
i \epsilon \partial_{t} u+\epsilon^{2} \Delta u-2 \lambda|u|^{p} u=0, x \in \mathbb{R}^{d}, t \in \mathbb{R}
$$

where $u$ is a complex valued function and $\epsilon \ll 1$ is a small dispersion parameter. Since $\epsilon$ has the same role as the reduced Planck constant $\hbar$ in the Schrödinger equation, the limit $\epsilon \rightarrow 0$ is also called the semiclassical limit in this context. The exponent $1<p<\infty$ represents the power of the non-linearity, and the parameter $\lambda= \pm 1$ determines if the non-linearity has a focusing $(\lambda=-1)$ or a defocusing $(\lambda=1)$ effect. The cubic $1+1$ dimensional NLS is a purely dispersive completely integrable equation with important applications in nonlinear optics and hydrodynamics [15]. Such dispersive partial differential equations can have solutions which develop a zone of rapid modulated oscillations in the region where the corresponding dispersionless equations have shocks or blow-up, i.e., a loss of regularity of the solution with respect to the initial data in finite time. Although an asymptotic description of these dispersive shocks is well known for certain integrable PDEs as the Korteweg-de Vries equation $[2,12,17]$ and the NLS equation for certain classes of initial data $[6,7,16]$, no such description is known for $(2+1)$-dimensional PDEs which, in addition, can have solutions which blow up. The appearance of blowup and the precise mechanism of it remain an open problem. For the time being, numerical simulations provide the only way to address these questions.

\footnotetext{
* I thank my supervisor C. Klein who interested me in the subject, for helpful discussions and writing corrections. This work benefited from financial support by the Conseil Régional de Bourgogne via a FABER grant allotted to the Pr. Klein, the ANR via the program ANR-09-BLAN-0117-01, and the Marie-Curie IRSES program RIMMP. This work was also granted access to HPC resources of CCRT/IDRIS under the allocation 2011106628 made by GENCI (Grand Equipement National de Calcul Intensif). In this context, I also thank B. Muite who contributed to the GENCI project.

1 Institut de Mathématiques de Bourgogne, Université de Bourgogne, 9 avenue Alain Savary, 21078 Dijon Cedex, France (kristelle.roidot@u-bourgogne.fr)
}

(C) EDP Sciences, SMAI 2012 
The Davey-Stewartson system can be written in the form

$$
\begin{array}{cl}
i \epsilon u_{t}+\epsilon^{2}\left(u_{x x}-\alpha u_{y y}\right)+2 \rho\left(\Phi+|u|^{2}\right) u & =0 \\
\Phi_{x x}+\alpha \Phi_{y y}+2|u|_{x x}^{2} & =0
\end{array}
$$

where $\alpha$ and $\rho$ take the values \pm 1 , where $\epsilon \ll 1$ is a small dispersion parameter, and where $\Phi$ is a mean field. The first equation is a nonlinear Schrödinger equation with a forcing term, and the second equation is a linear equation which is either elliptic or hyperbolic, in dependence of the sign of $\alpha$. These systems have been classified in accordance to this sign in [5] which corresponds to the type of the second order differential operators in both equations of the system. The hyperbolic-elliptic case $(\alpha=1)$ is known as DS II equation, and the DS I equation corresponds to the elliptic-hyperbolic case $(\alpha=-1)$. Both cases are completely integrable and can be seen as a higher dimensional generalization of the nonlinear Schrödinger equation (NLS). They appear for example in the description of gravity-capillarity wave packets in the limit of shallow water [1], [3], and also in plasma physics, to describe the propagation of plasma waves in strong magnetic fields $[13,14]$. In the following, we will only consider the case $\alpha=1$, since the mean field $\Phi$ is then obtained by inverting an elliptic operator.

A difficult problem in the numerical study of this equation is the so-called modulational instability, i.e., a self-induced amplitude modulation of a continuous wave propagating in a nonlinear medium, with subsequent generation of localized structures. It implies that high spatial resolution is needed for focusing NLS equations, see for instance [9]. Therefore, sequential codes used before, for example in [10] have to be parallelized to allow for such a spatial resolution (typically $2^{15} \times 2^{15}$ modes).

This paper is organized as follows: in section 1, we describe briefly the numerical difficulties related to the studied problem, and the solutions we adopted, in terms of numerical methods used and parallelization of codes. In section 2 we present numerical results and in section 3 we give concluding remarks.

\section{A simple PARALlEL SPECTRAL CODE}

\subsection{Numerical treatment}

Since critical phenomena are generally believed to be independent of the chosen boundary conditions, we consider a periodic setting. This includes rapidly decreasing functions which can be periodically continued within the finite numerical precision, as Schwartzian initial data one is interested in this paper. This allows to approximate the spatial dependence via truncated Fourier series which leads for the studied equations to large stiff systems of ODEs of the form

$$
v_{t}=\mathbf{L} v+\mathbf{N}(v, t)
$$

where $v$ denotes the (discrete) Fourier transform of $u$, and where $\mathbf{L}$ and $\mathbf{N}$ denote linear and nonlinear operators, respectively. The use of Fourier methods not only gives spectral accuracy in the spatial coordinates for smooth functions, but also minimizes the introduction of numerical dissipation which is important in the study of dispersive effects. In addition, it allows to deal with the problem of the modulational instability of the focusing DS II equation. The needed high resolution can be achieved by a parallel spectral code (see below) which essentially only requires existing highly optimized serial FFT algorithms. Such a code is very memory efficient in contrast to other approaches such as finite difference or finite element methods.

Systems studied in this work will be in general rather large and the PDEs are semilinear (i.e., high order derivatives are contained in the linear part $\mathbf{L}$ of (2), whereas the nonlinear part $\mathbf{N}$ contains no derivatives). This implies that the stiffness in these systems is due to the linear part. In [10], we did a comparison of fourth order time stepping schemes typically used to integrate stiff problems of the form (2). This allowed to identify Driscoll's method [4] as the best choice for the time integration of DS. This method is close to IMEX methods, which use a stable implicit method for the linear part of the equation (2) and an explicit scheme for the nonlinear part which is assumed to be non-stiff. Driscoll's [4] more sophisticated variant splits also the linear part of the equation in Fourier space into regimes of high and low frequencies, and uses the fourth order Runge-Kutta (RK) 
integrator for the latter and the linear implicit RK method of order three for the former regime. In [8], IMEX schemes did not perform satisfactorily for dispersive PDEs in contrast to Driscoll's method which provides an efficient tool in this context $[9,10]$. We thus implement this scheme in FORTRAN 90, using MPI library for the parallelization, briefly described below.

\subsection{Parallelization}

A prerequisite for parallel numerical algorithms is that sufficient independent computations can be identified for each processor, that require only small amounts of data to be communicated between periods of independent computations. To this end, we do a data decomposition, which makes it possible to do basic operations on each object in the data domain (vector, matrix...) to be executed safely in parallel by the available processors.

Our domain decomposition is implemented by developing a code describing the local computations and local data structures for a single process.

Global arrays are divided in the following way: denoting by $x_{n}=-\pi L_{x}+\frac{2 \pi(n-1) L_{x}}{N_{x}-1}$, with $n=1 \ldots N_{x}$, and $y_{m}=-\pi L_{y}+\frac{2 \pi(m-1) L_{y}}{N_{y}-1}$, with $m=1 \ldots N_{y}$, the respective discretizations of $x$ and $y$ in the corresponding computational domain $x \times y \in\left[-L_{x} \pi, L_{x} \pi\right] \times\left[-L_{y} \pi, L_{y} \pi\right], u$ is then represented by a $N_{x} \times N_{y}$ matrix. For programming ease and for the efficiency of the Fourier transform, $N_{x}$ and $N_{y}$ are chosen to be powers of two. The number of MPI processes, $n_{p}$ is chosen to divide $N_{x}$ and $N_{y}$ perfectly, so that each processor holds $N_{x} \times N_{y} / n_{p}$ elements of $u$, for example processor $i$ holds the elements

$$
u\left(1: N_{x},(i-1) \cdot \frac{N_{y}}{n_{p}}+1: i \cdot \frac{N_{y}}{n_{p}}\right)
$$

in the global array and then each parallel task then works on a portion of the data.

While processors execute an operation, they may need values from other processors. The above domain decomposition has been chosen such that the distribution of operations is balanced and that the communication is minimized. More precisely, the only part of our code which requires communications is the computation of the 2d-FFT. To avoid performing global Fourier transforms which are inefficient, the array is transposed once all the one dimensional Fourier transforms in the $x$ direction have been done (using FFTW 3.21 library, which is close to optimal on $\mathrm{x} 86$ architectures and allows the resulting program to be portable but still simple). Since the data is evenly distributed among the MPI processes, the transpose is then efficiently implemented using MPI_ALLTOALL. Computations on the global arrays also have been transformed, e.g., by adapting the loop bounds, to ensure that only local array elements are computed. The access to remote elements has been implemented via explicit communications, using sub-routines of the MPI library, temporary variables have been allocated, messages have been constructed and transmitted to the target process.

\section{Numerical Results}

We consider the focusing DS II equation in the semiclassical limit $(\epsilon \rightarrow 0)$, and consider initial data of the form

$$
u_{0}(x, y)=e^{-R^{2}}, \text { with } R=\sqrt{x^{2}+\eta y^{2}}
$$

The computation is carried out with $2^{15} \times 2^{15}$ points for $x \times y \in[-5 \pi, 5 \pi] \times[-5 \pi, 5 \pi]$, and with a time step size $\Delta_{t}=3 e^{-4}$, for different values of $\eta$, and $\epsilon=0.1$. This resolution allows to get machine precision in the decay of the Fourier coefficients, and to avoid numerical difficulties related to the modulational instability. The maximal time of computation is chosen to be $t_{\max }=0.6$, as twice the apparent break up time of the corresponding dispersionless DS system.

For $\eta=0.1$, the initial peak grows until its maximal height at $t \sim 0.3$. After that smaller humps appear, see Fig. [1 as in the case of the one dimensional cubic NLS equation in semiclassical limit. A similar behavior is observed for $\eta=0.3$, see Fig. [2] The Fourier coefficients at $t_{\max }$ are shown in Fig. [3 for the case $\eta=0.1$. They decrease to machine precision, and do not show any sign of modulational instability, which means that 

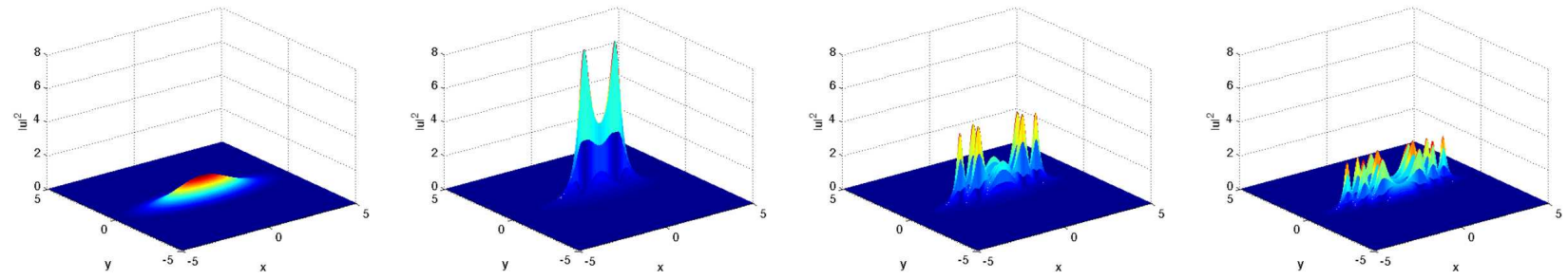

Figure 1. Modulus of the solution to the focusing DS II equation for initial data of the form (4) with $\eta=0.1$, for $t=0.15, t=0.3, t=0.45$, and $t=0.6$.
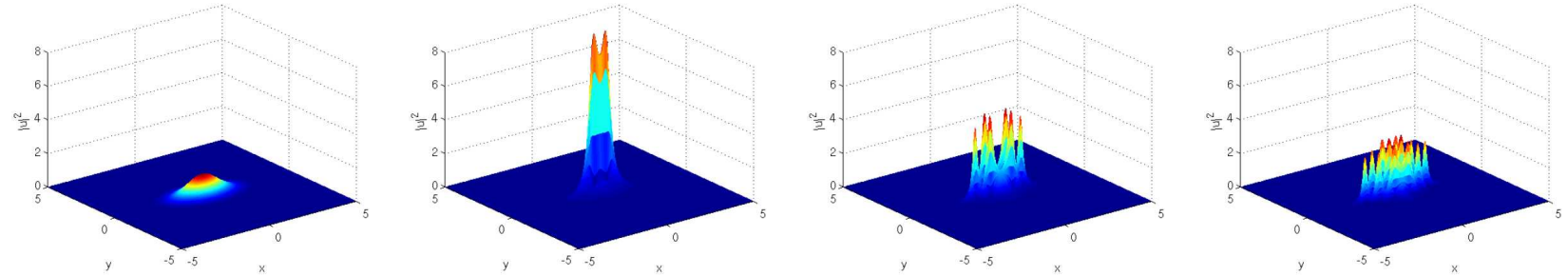

FIgURE 2. Modulus of the solution to the focusing DS II equation for initial data of the form (44) with $\eta=0.3$, for $t=0.15, t=0.3, t=0.45$, and $t=0.6$.

the solution is well resolved. The same applies for the case $\eta=0.3$.
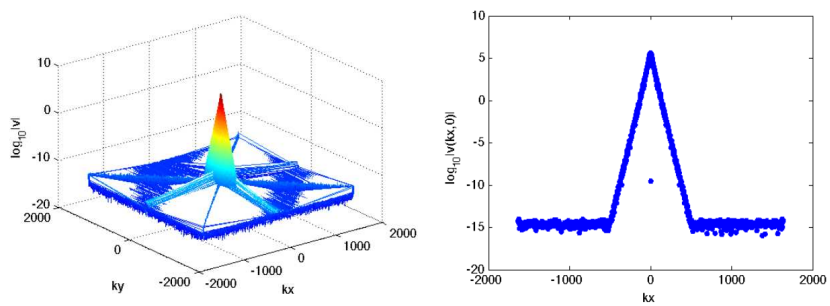

FiguRE 3. Fourier Coefficients of $u$ at $t_{\max }=0.6$ for initial data of the form (4) with $\eta=0.1$.

For $\eta=0.5$ and $\eta=0.8$, we observe three peaks of the solution, again followed by the appearance of smaller humps, see Fig. [4 Fig. 5 and 7 The Fourier coefficients at $t_{\max }$ are shown in fig. [6 for the case $\eta=0.5$
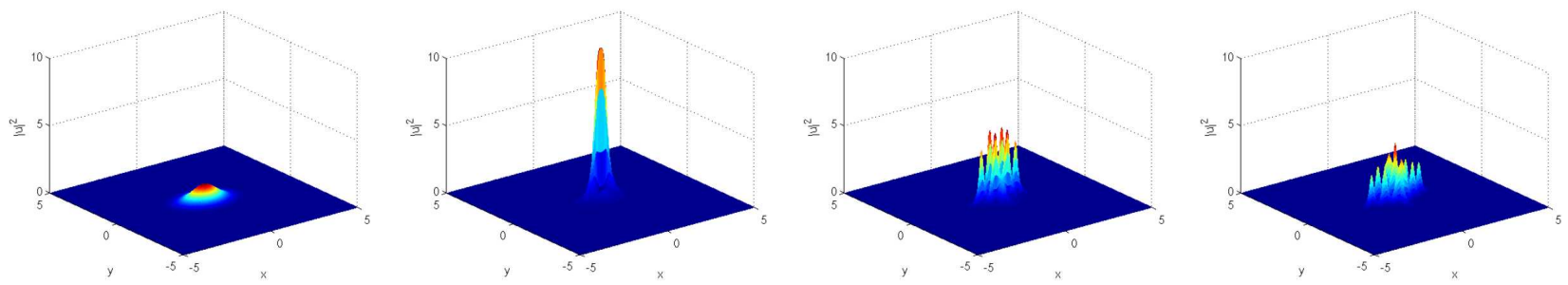

FIgURE 4. Modulus of the solution to the focusing DS II equation for initial data of the form (4) with $\eta=0.5$, for $t=0.15, t=0.3, t=0.45$, and $t=0.6$. 

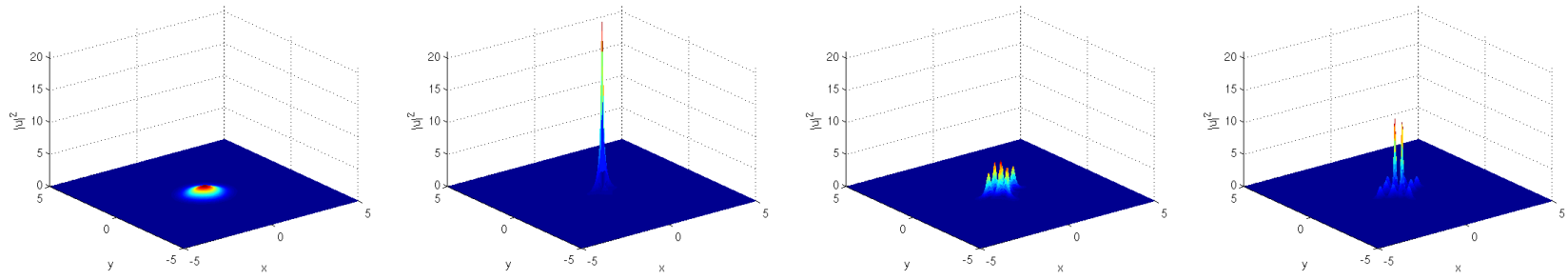

Figure 5. Modulus of the solution to the focusing DS II equation for initial data of the form (4) with $\eta=0.8$, for $t=0.15, t=0.3, t=0.45$, and $t=0.6$.

and for $\eta=0.8$. They again decrease to machine precision. We show in Fig. 7 the evolution of $\max \left(|u|^{2}\right)$ in dependence of time and the contour of the solution at the final time of computation $\left(t_{\max }=0.6\right)$ for the values of $\eta$ discussed before.
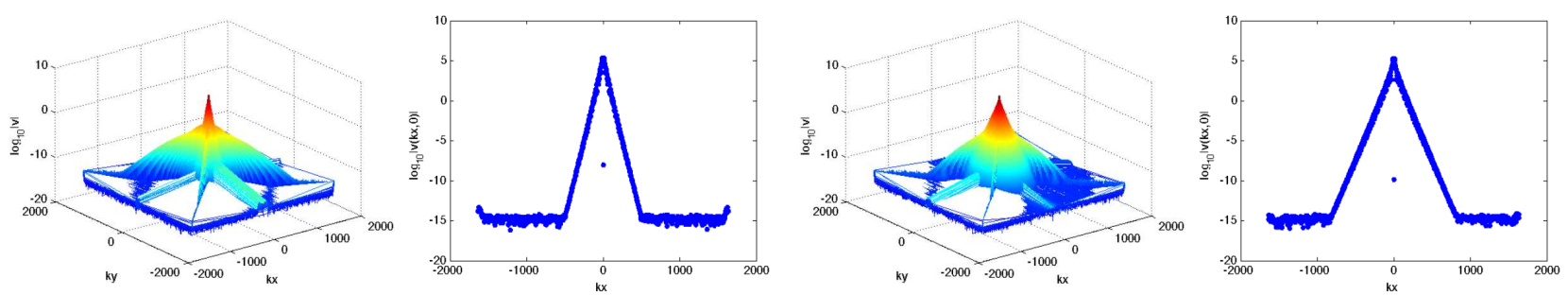

Figure 6. Fourier Coefficients of $u$ at $t_{\max }=0.6$ for initial data of the form (4) with $\eta=0.5$ (left) and $\eta=0.8$ (right).
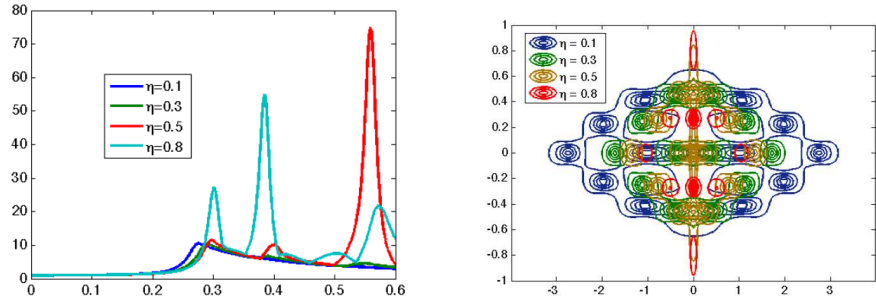

Figure 7. Time evolution of $\max \left(|u|^{2}\right)$ and contours of the solution at $t_{\max }$ for different $\eta$ for initial data of the form (4).

For $\eta=1$, the code breaks at $t \sim 0.28$, after the apparition of a blow up. As discussed in [11], a jump in the numerically computed energy, i.e., a conserved quantity of DS appears at this time. The latter is given by

$E[u(t)]:=\frac{1}{2} \int_{\mathbb{T}^{2}}\left[\epsilon^{2}\left|\partial_{x} u(t, x, y)\right|^{2}-\epsilon^{2}\left|\partial_{y} u(t, x, y)\right|^{2}-\rho\left(|u(t, x, y)|^{4}-\frac{1}{2}\left(\Phi(t, x, y)^{2}+\left(\partial_{x}^{-1} \partial_{y} \Phi(t, x, y)\right)^{2}\right)\right)\right] d x d y$,

and we consider

$$
\Delta_{E}=\left|1-\frac{E(t)}{E(0)}\right| \text {. }
$$

We show this jump and the time evolution of $\max \left(|u|^{2}\right)$ in Fig. 8 

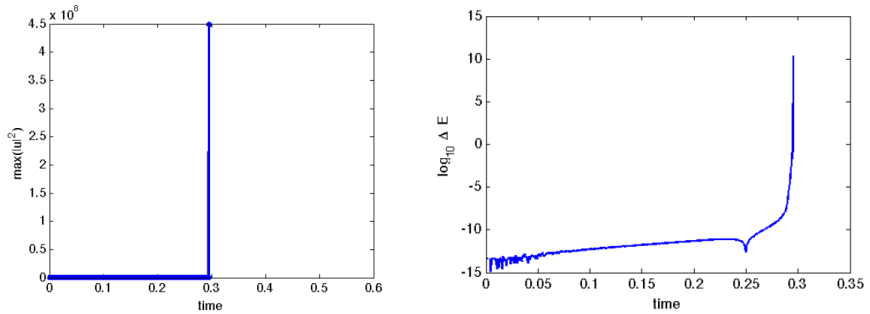

Figure 8. Time evolution of $\max \left(|u|^{2}\right)$ and of $\Delta_{E}(t)$ for initial data of the form (4) with $\eta=1$.

\section{Conclusion}

It was shown in this work that parallel computing is an powerful tool nowadays for numerical simulations of problems yet inaccessible for a theoretical treatment. The writing of parallelized code in Fortran 90 allowed to perform a valid numerical study of the focusing DS II equation in the semiclassical limit. We showed that a blow up occurs for initial data with radial symmetry, as in the critical one-dimensional case (in which the nonlinearity is quintic), whereas more general initial data lead to solutions with dispersive shocks.

\section{REFERENCES}

[1] A. Davey and K. Stewartson. On Three-dimensional Packets of Surface Waves. Proc. R. Soc. Lond. A., 338:101-110, 1974.

[2] P. Deift, S. Venakides, and X. Zhou. New Result in small Dispersion KdV by an Extension of the steepest Descent Method for Riemann-Hilbert Problems. Comm. Pure Appl. Math., 38:125-155, 1985.

[3] V.D. Djordjevic and L.G. Redekopp. On Two-dimensional Packets of Capillarity-Gravity Waves. J. Fluid Mech., 79 part 4:703-714, 1977.

[4] T.A. Driscoll. A composite Runge-Kutta Method for the spectral Solution of semilinear PDEs. Journal of Computational Physics, 182:357-367, 2002.

[5] J-M Ghidaglia and J-C Saut. On the initial Value Problem for the Davey-Stewartson Systems. Nonlinearity, 3, 1990.

[6] S. Jin, C.D. Levermore, and D.W. McLaughlin. The Behavior of Solutions of the NLS Equation in the semiclassical Limit. In Singular Limits of Dispersive Waves, 1994.

[7] S. Kamvissis, K.D.T.-R. McLaughlin, and P.D. Miller. Semiclassical Soliton Ensembles for the focusing nonlinear Schrödinger Equation. Princeton University Press, 2003.

[8] A-K. Kassam and L.N. Trefethen. Fourth-Order Time-Stepping for stiff PDEs. SIAM J. Sci. Comput., 26(4):1214-1233, 2005.

[9] C. Klein. Fourth-Order Time-Stepping for low Dispersion Korteweg-de Vries and nonlinear Schrödinger Equation. Electronic Transactions on Numerical Analysis., 39:116-135, 2008.

[10] C. Klein and K. Roidot. Fourth order time-stepping for Kadomtsev-Petviashvili and Davey-Stewartson equations. SIAM J. Sci. Comp., 2011.

[11] C. Klein, K. Roidot, and B. Muite. Numerical Study of Blowup in the Davey-Stewartson System. Preprint in preparation, 2011.

[12] P.D. Lax and C.D. Levermore. The small Dispersion Limit of the Korteweg de Vries Equation I, II, III. Comm. Pure Appl. Math., 36:253-290, 571-593, 809-830, 1983.

[13] K. Nishinari, K. Abe, and J. Satsuma. A new Type of Soliton Behaviour in a Two dimensional Plasma System. J. Phys. Soc. Jpn., 62:2021-2029, 1993.

[14] K. Nishinari, K. Abe, and J. Satsuma. Multidimensional Behavior of an electrostaic Ion Wave in a magnetized Plasma. Phys. Plasmas, 1:2559-2565, 1994.

[15] C. Sulem and P.L. Sulem. The nonlinear Schrödinger Equation. Springer, 1999.

[16] A. Tovbis, S. Venakides, and X. Zhou. On semiclassical (Zero Dispersion Limit) Solutions of the focusing nonlinear Schrödinger Equation. Communications on pure and applied mathematics, 57(7):877-985, 2004.

[17] S. Venakides. The Zero Dispersion Limit of the Korteweg de Vries Equation for initial Potential with nontrivial Reflection Coefficient. Comm. Pure Appl. Math., 38:125-155, 1985. 